\title{
Probing nuclear effects with neutrino-induced charged-current neutral pion production
}

D. Coplowe, ${ }^{1}$ O. Altinok, ${ }^{2}$ Z. Ahmad Dar, ${ }^{3}$ F. Akbar, ${ }^{3}$ D. A. Andrade,${ }^{4}$ G. D. Barr, ${ }^{1}$ A. Bashyal, ${ }^{5}$ A. Bercellie,${ }^{6}$ M. Betancourt, ${ }^{7}$ A. Bodek, ${ }^{6}$ A. Bravar, ${ }^{8}$ H. Budd,${ }^{6}$ G. Caceres, ${ }^{9}$ T. Cai, ${ }^{6}$ M. F. Carneiro, ${ }^{5,9, \dagger}$ H. da Motta, ${ }^{9}$ S. A. Dytman, ${ }^{10}$ G. A. Díaz, ${ }^{6,11}$ J. Felix, ${ }^{4}$ L. Fields, ${ }^{7}$ A. Filkins, ${ }^{12}$ R. Fine, ${ }^{6}$ A. M. Gago, ${ }^{11}$ H. Gallagher, ${ }^{2}$ A. Ghosh, ${ }^{13,9}$ R. Gran, ${ }^{14}$ D. A. Harris, ${ }^{15,7}$ S. Henry, ${ }^{6}$ S. Jena ${ }^{16}{ }^{16}$ J. Kleykamp, ${ }^{6}$ M. Kordosky ${ }^{12}$ D. Last, ${ }^{17}$ T. Le, ${ }^{2,18}$ A. Lozano, ${ }^{9}$ X.-G. Lu® ${ }^{1, *}$ E. Maher, ${ }^{19}$ S. Manly, ${ }^{6}$ W. A. Mann, ${ }^{2}$ C. Mauger,${ }^{17}$ K. S. McFarland, ${ }^{6}$ B. Messerly,${ }^{10}$ J. Miller, ${ }^{13}$ J. G. Morfín, ${ }^{7}$ D. Naples ${ }^{10}$ J. K. Nelson, ${ }^{12}$ C. Nguyen, ${ }^{20}$ A. Norrick, ${ }^{12}$ A. Olivier, ${ }^{6}$ V. Paolone,${ }^{10}$ G. N. Perdue, ${ }^{7,6}$ M. A. Ramírez, ${ }^{4}$ R. D. Ransome, ${ }^{18}$ H. Ray, ${ }^{20}$ P. A. Rodrigues, ${ }^{1,21,6}$ D. Ruterbories, ${ }^{6}$ H. Schellman, ${ }^{5}$ J. T. Sobczyk, ${ }^{22}$ C. J. Solano Salinas, ${ }^{23}$

H. Su, ${ }^{10}$ M. Sultana, ${ }^{6}$ V. S. Syrotenko, ${ }^{2}$ E. Valencia, ${ }^{12,4}$ D. Wark, ${ }^{1}$ A. Weber, ${ }^{1}$ M. Wospakrik, ${ }^{20}$ C. Wret, ${ }^{6}$ B. Yaeggy, ${ }^{13}$ and L. Zazueta ${ }^{12}$

(MINER $\nu$ A Collaboration)

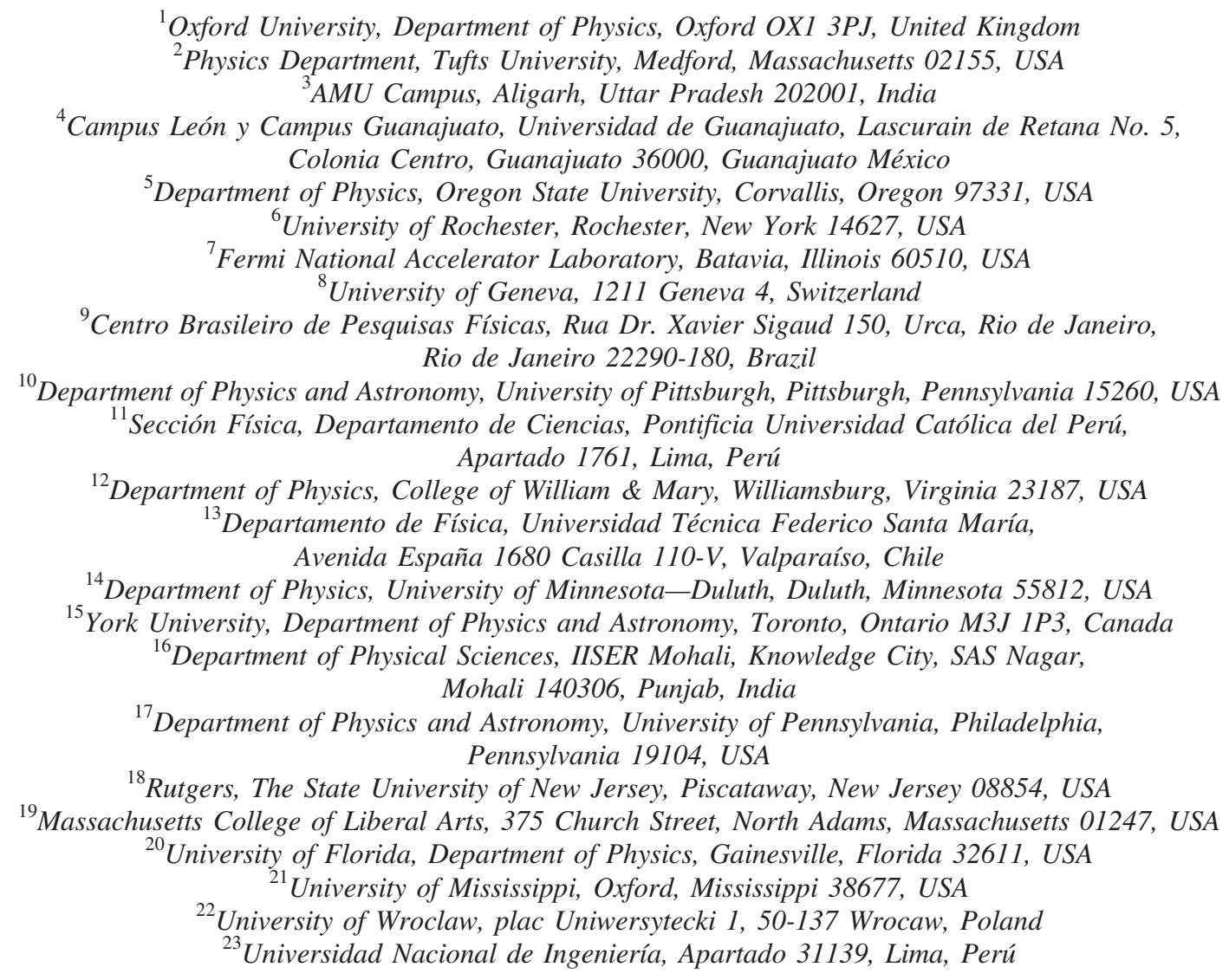

(Received 13 February 2020; accepted 28 September 2020; published 19 October 2020; corrected 11 December 2020)

We study neutrino-induced charged-current $(\mathrm{CC}) \pi^{0}$ production on carbon nuclei using events with fully imaged final-state proton- $\pi^{0}$ systems. Novel use of final-state correlations based on transverse kinematic

\footnotetext{
*Corresponding author. xianguo.lu@physics.ox.ac.uk

${ }^{\dagger}$ Present address: Brookhaven National Laboratory, Upton, NY 11973, USA.
}

Published by the American Physical Society under the terms of the Creative Commons Attribution 4.0 International license. Further distribution of this work must maintain attribution to the author(s) and the published article's title, journal citation, and DOI. Funded by $S C O A P^{3}$. 
imbalance enables the first measurements of the struck nucleon's Fermi motion, of the intranuclear momentum transfer (IMT) dynamics, and of the final-state hadronic momentum configuration in neutrino pion production. Event distributions are presented for (i) the momenta of neutrino-struck neutrons below the Fermi surface, (ii) the direction of missing transverse momentum characterizing the strength of IMT, and (iii) proton-pion momentum imbalance with respect to the lepton scattering plane. The observed Fermi motion and IMT strength are compared to the previous MINER $\nu \mathrm{A}$ measurement of neutrino $\mathrm{CC}$ quasielastic-like production. The measured shapes and absolute rates of these distributions, as well as the cross section asymmetries, show tensions with predictions from current neutrino generator models.

DOI: 10.1103/PhysRevD.102.072007

\section{INTRODUCTION}

In high-statistics neutrino oscillation experiments, the measurement precision of the fundamental properties of neutrinos is becoming limited by knowledge of neutrinonucleus interactions [1]. The nuclear medium introduces deviations from free-nucleon scattering that are poorly known and are leading sources of systematic uncertainty for measurements of the $\mathcal{C} \mathcal{P}$-violating phase [2,3]. Among the prominent interaction modes produced when studying neutrino flavor transformation are final states containing pions. For future experiments such as DUNE [4] and HyperKamiokande [5] to achieve their designed sensitivity, it is imperative to assess the various nuclear effects involved in pion production.

In previous studies, MINER $\nu \mathrm{A}$ measured the final-state correlations in neutrino charged-current (CC) quasielastic (QE)-like interactions on carbon [6,7]. These correlations are based on transverse kinematic imbalance (TKI) that helps precisely identify intranuclear dynamics [6-15] or the absence thereof [16-20]. The previously measured imbalance is between the CC muon and the final-state proton; the present work studies the following reaction including additional neutral pions in the final state:

$$
\nu_{\mu}+\mathrm{C} \rightarrow \mu^{-}+\underbrace{\mathrm{p}+\pi^{0}}_{\mathrm{h}}+\mathrm{X}
$$

where $\mathrm{X}$ is a final-state hadronic system consisting of the nuclear remnant with possible additional protons and neutral pions but without other mesons. The only difference in the kinematics here is to replace the previous proton final state by $\mathrm{h}$ [Eq. (1)] that is the combination of the proton and neutral pion. Therefore, the transverse boosting angle, $\delta \alpha_{\mathrm{T}}$ [8], and the emulated nucleon momentum, $p_{\mathrm{n}}[9,13]$, are defined similarly as in Ref. [6],

$$
\begin{gathered}
\vec{p}_{\mathrm{h}}=\vec{p}_{\mathrm{p}}+\vec{p}_{\pi^{0}}, \\
\delta \vec{p}_{\mathrm{T}}=\vec{p}_{\mathrm{T}}^{\mu}+\vec{p}_{\mathrm{T}}^{\mathrm{h}}, \\
\delta \alpha_{\mathrm{T}}=\arccos \frac{-\vec{p}_{\mathrm{T}}^{\mu} \cdot \delta \vec{p}_{\mathrm{T}}}{p_{\mathrm{T}}^{\mu} \delta p_{\mathrm{T}}},
\end{gathered}
$$

$$
\begin{gathered}
\delta p_{\mathrm{L}}=\frac{1}{2} R-\frac{m_{\mathrm{C}^{\prime}}^{2}+\delta \vec{p}_{\mathrm{T}}^{2}}{2 R}, \quad \text { with } \\
R \equiv m_{\mathrm{C}}+p_{\mathrm{L}}^{\mu}+p_{\mathrm{L}}^{\mathrm{h}}-E_{\mu}-E_{\mathrm{h}}, \text { and finally } \\
p_{\mathrm{n}}=\sqrt{\delta p_{\mathrm{T}}^{2}+\delta p_{\mathrm{L}}^{2}}
\end{gathered}
$$

where $p_{\kappa}$ is the momentum of particle $\kappa$. The subscripts T and $\mathrm{L}$ stand for transverse and longitudinal components with respect to the neutrino direction (Fig. 1). $\delta \vec{p}_{\mathrm{T}}$ is the missing transverse momentum, or missing $\mathrm{pT}$, between the final state $\mu+\mathrm{h}$ and the initial state $\nu_{\mu}$; its longitudinal counterpart, $\delta p_{\mathrm{L}}$, depends on the muon and hadron energies, $E_{\mu}$ and $E_{\mathrm{h}}$, and the carbon-nucleus mass, $m_{\mathrm{C}^{(\prime)}}$, before (after) the interaction,

$$
m_{\mathrm{C}^{\prime}}=m_{\mathrm{C}}-m_{\mathrm{n}}-b,
$$

where $m_{\mathrm{n}}$ is the neutron mass. The excitation energy $b$ is $+28.7 \mathrm{MeV}[9,13]$, which affects $m_{\mathrm{C}^{\prime}}$ only at a per mil level and therefore has a negligible systematic impact.

The transverse boosting angle, $\delta \alpha_{\mathrm{T}}$ [Eq. (4)], describes the angular deviation of $\delta \vec{p}_{\mathrm{T}}$ from the transverse momentum transfer, $-\vec{p}_{\mathrm{T}}^{\mu}$. The configuration $\delta \alpha_{\mathrm{T}}=0$ is only

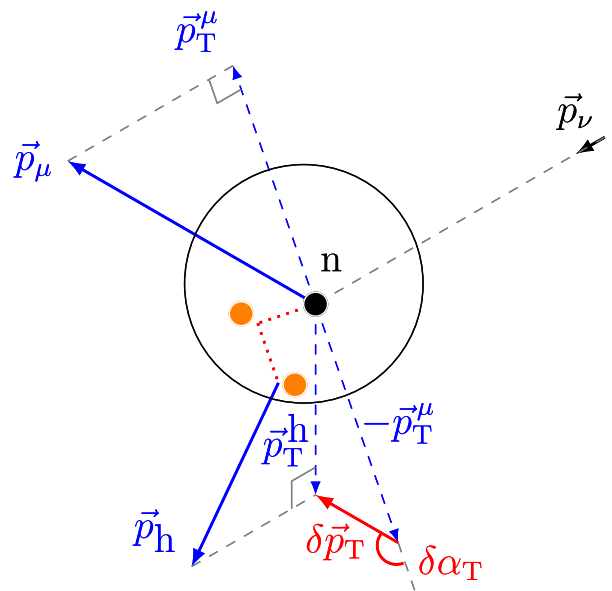

FIG. 1. Schematic diagram of the single-transverse kinematics [8]. 
possible due to Fermi motion. Because Fermi motion is isotropic, events subject to Fermi motion and no other nuclear effects are evenly distributed in $\delta \alpha_{\mathrm{T}}$. Processes with intranuclear momentum transfer (IMT), such as final-state interactions (FSIs), pion absorption by the remnant, and production of extra particles included in $\mathrm{X}$, will increase $\delta \alpha_{\mathrm{T}}$; the resulting nonflatness in the $\delta \alpha_{\mathrm{T}}$ distribution indicates how much impedance the outgoing hadrons experience inside the nucleus $[6,8,10]$.

Following Eq. (7), it can be shown using a general event generator that $p_{\mathrm{n}}$ is approximated by $\delta p_{\mathrm{T}}$ with an $\mathcal{O}(10 \%)$ correction. On the one hand, when X is carbon-11 in Eq. (1) and the final-state proton and neutral-pion do not experience FSIs, $p_{\mathrm{n}}$ is the initial momentum of the struck neutron in the resonant production,

$$
\nu_{\mu}+\mathrm{n} \rightarrow \mu^{-}+\mathrm{p}+\pi^{0} .
$$

On the other hand, in the presence of IMT, $\delta p_{\mathrm{T}}$ increases due to the extra momentum lost by FSIs or the unaccounted-for momentum share with other final-state particles in $\mathrm{X}$, leading to a higher value of $p_{\mathrm{n}}$ beyond the Fermi surface.

The initial neutron Fermi motion and IMT in QE-like interactions, including also two-particle-two-hole (2p2h) contributions, have been measured and discussed in Ref. [6]. This paper reports the first measurement of TKI(-based) final-state correlations, $\delta \alpha_{\mathrm{T}}$ and $p_{\mathrm{n}}$, in pion production. The measured Fermi motion and IMT dynamics will be compared to the QE-like measurement.

In addition to studying the hadronic final state as a whole, the proton and pion final states can be compared against each other to gain new insights into the hadronic momentum configuration in pion production as follows: the double-transverse momentum imbalance, $\delta p_{\mathrm{TT}}$, describes the momentum imbalance of the final-state hadrons along the axis that is perpendicular to the lepton scattering plane [16],

$$
\begin{gathered}
\delta p_{\mathrm{TT}}=\delta \vec{p}_{\mathrm{T}} \cdot \hat{z}_{\mathrm{TT}} \\
=\vec{p}_{\mathrm{h}} \cdot \hat{z}_{\mathrm{TT}},
\end{gathered}
$$

where $\hat{z}_{\mathrm{TT}}$ is a unit vector along $\vec{p}_{\nu} \times \vec{p}_{\mu}$ (Fig. 2). In the absence of nuclear effects, $\delta p_{\mathrm{T}}$ vanishes and so does $\delta p_{\mathrm{TT}}$, whereas for nuclei with $\mathrm{A}>1$, nonzero contributions arise from the nuclear medium. Because the proton and pion spatial distributions in resonant production might be asymmetric with respect to the lepton scattering plane (cf. Ref. [15] and references therein), any particle-typedependent nuclear effects could cause an asymmetric distribution of $\delta p_{\mathrm{TT}}$. This paper presents the first measurement of this new TKI.

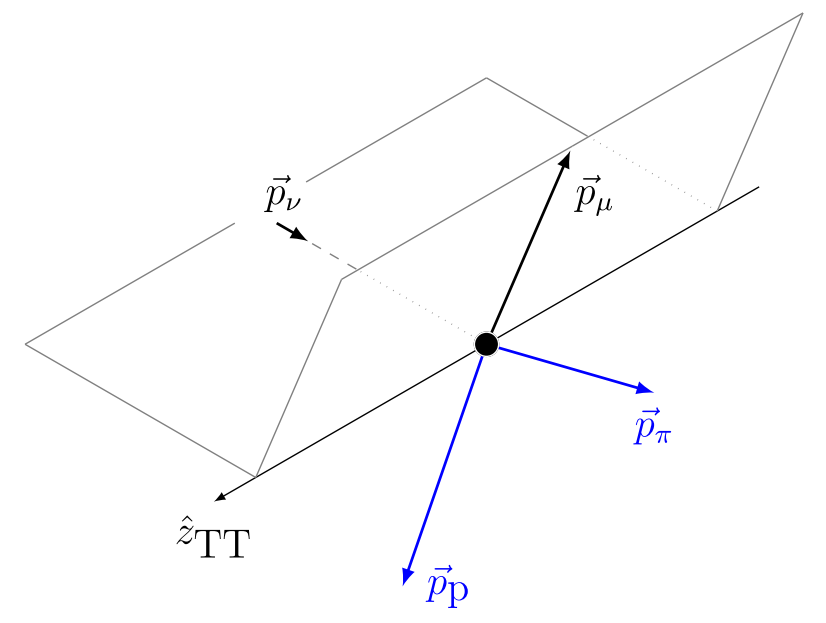

FIG. 2. Schematic diagram of the double-transverse kinematics [16]. The neutrino and muon momenta define the double-transverse axis $\hat{z}_{\mathrm{TT}} \equiv \vec{p}_{\nu} \times \vec{p}_{\mu} /\left|\vec{p}_{\nu} \times \vec{p}_{\mu}\right|$. The scattering plane is spanned by the lepton momentum vectors and therefore perpendicular to $\hat{z}_{\mathrm{TT}}$.

\section{MEASUREMENT}

In the present work, the signal is defined as $\mathrm{CC} \nu_{\mu}$ events on carbon whose final state is $1 \mu^{-} N \mathrm{p} M \pi^{0}(N, M>0)$ with any number of neutrons and no other particles exiting the nucleus [Eq. (1)]. Kinematic constraints, resulting from detector acceptance and response, are placed on the final state satisfying

$$
\begin{gathered}
1.5 \leq p_{\mu}(\mathrm{GeV} / c) \leq 20.0, \quad \theta_{\mu}<25^{\circ}, \\
p_{\mathrm{p}} \geq 0.45 \mathrm{GeV} / c
\end{gathered}
$$

where $\theta_{\mu}$ is the muon polar angle with respect to the neutrino direction. The momenta of the leading proton and $\pi^{0}$ are used in the calculation of the observables.

The analysis uses data obtained with the MINER $\nu \mathrm{A}$ detector exposed to the NuMI low energy neutrino beam $\left(\left\langle E_{\nu}\right\rangle=3 \mathrm{GeV}\right)$ with $3.33 \times 10^{20}$ protons on target (POT). The total neutrino flux $\left(2.88 \times 10^{-8} / \mathrm{cm}^{2} / \mathrm{POT}\right)$ is estimated according to Ref. [21]. Neutrino interactions inside a fiducial volume within MINER $\nu$ A's active tracker with a mass of 5.3 tons are selected. Precise tracking is achieved inside this fiducial volume by an alternating arrangement of hexagonal plastic scintillator planes at $0^{\circ}$ and $\pm 60^{\circ}$ to the vertical. Each plane consists of 127 triangular polystyrene (CH) strips up to $245 \mathrm{~cm}$ long, $1.7 \mathrm{~cm}$ in height, and a width of $3.3 \mathrm{~cm}$. The strips form a plane by alternating strips such that the cross sectional view is a regular trapezium. Located two meters downstream of MINER $\nu \mathrm{A}$ is the MINOS near detector-a magnetized muon spectrometer used to measure both the charge and momentum of muons. A detailed description of both detectors can be found in Refs. [22,23]. 
Neutrino events are simulated in the detector using GENIE 2.8.4 [24] where the initial state is modeled as a relativistic global Fermi gas (RFG) [25]. GENIE describes CC QE processes following Ref. [26] with a dipole axial mass $\left(M_{\mathrm{A}}^{\mathrm{QE}}\right)$ of $0.99 \mathrm{GeV}$ [27]. The production of $\Delta$ and higher resonances used the Rein-Sehgal single pion model [28]. Nonresonant pion production and multipion resonance contributions are introduced with a GENIE-specific model [29]. This background component is simulated up to a hadronic invariant mass range of $W<1.7 \mathrm{GeV}$ [30-32]. All resonant baryons decay isotropically in their rest frame with the exception of the $\Delta^{++}$. Following [33], the $\Delta^{++}$ angular isotropy is suppressed by $50 \%$ of that predicted by Rein-Sehgal. Deep inelastic scattering (DIS) is incorporated into GENIE via the 2003 Bodek-Yang model [34] and hadronization is described by PYTHIA6 [35] and models based on Koba-Nielsen-Olesen scaling [36,37].

GENIE's default simulation is augmented to incorporate recent developments in both theory and experimental results as follows: $2 \mathrm{p} 2 \mathrm{~h}$ contributions based on the Valencia model [38-41] are included; the relative strength has been scaled upward in accord with the MINER $\nu$ A lowrecoil measurement [42]. Long-range correlations are included in QE interactions via the random phase approximation [43]. Finally, a reduction of 53\% in GENIE's nonresonant single-pion prediction is applied in accord with recent analyses of deuterium bubble chamber data $[31,32]$. GENIE applies an effective model of FSI based on Ref. [44].

The propagation of final-state particles within the detector is simulated using GEANT4 9.4.2 [45]. Hadron test beam data provided by a scaled down version of MINER $\nu \mathrm{A}$ are used to constrain the GEANT4 simulation of protons and charged pions [22]. For both data and simulation, the energy scale is calibrated using through-going muons. These procedures ensure that the energy deposited per plane agrees between data and simulation.

Signal-like events are selected by first requiring a single track originating from within MINER $\nu$ A's tracker to match a negatively charged track identified by MINOS. This track must fulfill the kinematic constraints outlined in Eq. (12). The muon's starting position, or primary vertex, is assessed for the existence of any additional tracks. In instances where extra tracks exist, the primary vertex is redetermined to account for the extra information provided by these tracks. All nonmuon tracks are required to be protonlike by comparing their measured $\mathrm{d} E / \mathrm{d} x$ profiles to the simulated ones for protons and charged pions. Only protonlike tracks are retained and their ranges are used to determine their momenta. The leading proton must pass the phase-space requirement from Eq. (13).

Neutral pions are identified from their dominant decay signature, $\pi^{0} \rightarrow \gamma \gamma$, by requiring exactly two electromagnetic showers. Their direction must be consistent with originating from the primary vertex. The calorimetric

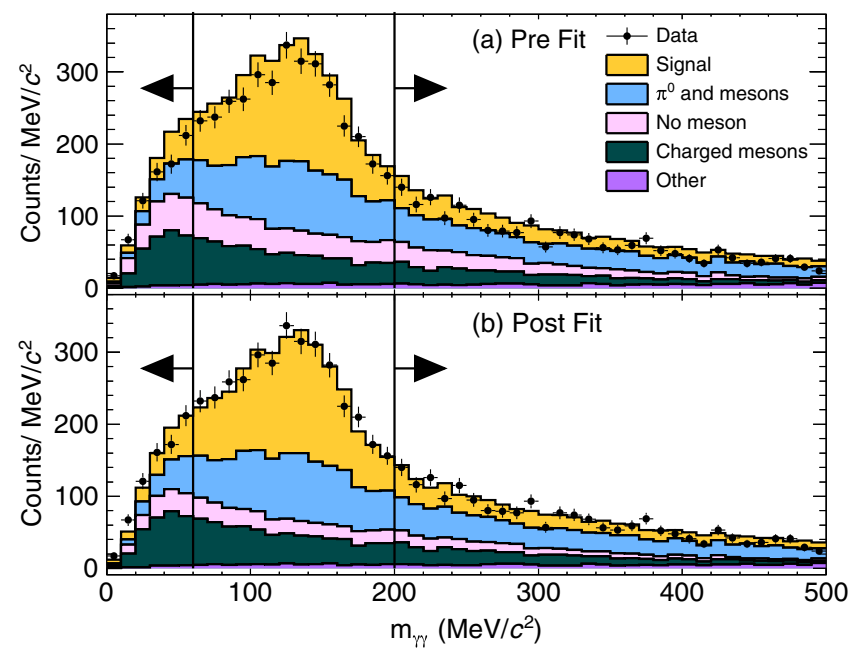

FIG. 3. Reconstructed distributions of the two-photon invariant mass in the selected sample, compared to simulations (a) before and (b) after the background fit. For completeness, the excluded regions are also shown, indicated by the arrows. The Other category contains $1 \mu^{-} N \mathrm{p} M \pi^{0}(N, M>0)$ events which are out of acceptance.

energy and direction of both photons are combined to reconstruct the $\pi^{0}$ 's momentum. Any remaining charged pion background is reduced by requiring that no Michel electronlike signature (indicating the presence of final-state $\pi^{+} \mathrm{s}$ ) exists in the candidate events. The signal purity is improved by reconstructing the invariant mass, $m_{\gamma \gamma}$, of the two photons using

$$
m_{\gamma \gamma}=\sqrt{2 E_{1} E_{2}\left(1-\cos \theta_{12}\right)},
$$

where $E_{1}$ and $E_{2}$ are the photon energies, and $\theta_{12}$ is the opening angle between the two photons. Signal events are required to be within $60 \leq m_{\gamma \gamma}(\mathrm{MeV} / c) \leq 200$. Full details of the selection can be found in previous MINER $\nu \mathrm{A}$ measurement [46] which, however, placed an upper bound on the (experimental) hadronic invariant mass $W$ at $1.8 \mathrm{GeV} / c^{2}$ and required there be one and only one $\pi^{0}$ regardless of the protons in the final state.

The resulting sample has $51.4 \%$ purity and 5.7\% efficiency. For a neutrino-proton interaction, the $1 \mu^{-} N \mathrm{p} M \pi^{0}(N, M>0)$ final-state requirement only allows multiproton production which is highly suppressed. Therefore, almost all events from the hydrogen component of the $\mathrm{CH}$ target contribute to the background. Overall, the dominant background categories are (A) $\pi^{0}$ events with other mesons ( $\pi^{0}$ and mesons), (B) events without $\pi^{0} \mathrm{~s}$ (charged mesons), and (C) zero-meson events (no meson), in decreasing order of importance, as is shown in Fig. 3. A data-driven approach is used to constrain these background components. Three sidebands are obtained by losing one of the signal selection criteria: one sideband utilized events below and above the $m_{\gamma \gamma}$ range allowed to 

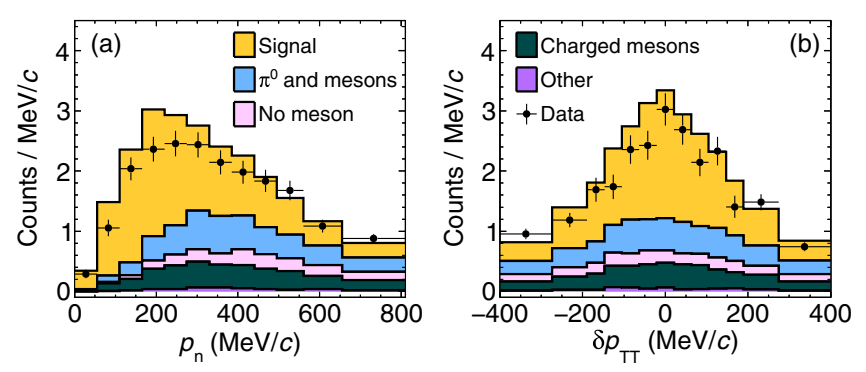

FIG. 4. Reconstructed distributions of (a) $p_{\mathrm{n}}$ and (b) $\delta p_{\mathrm{TT}}$, compared to simulations after the background fit.

the selected sample, the second one used events that fail the quality requirements for proton tracks, the third one used events that were accompanied by a Michel electron tag. The size of the backgrounds (A)-(C) is tuned to describe the data in these sidebands. The resulting scaling factors are $0.92,1.12$, and 0.67 , respectively. Compared to Ref. [46], all the backgrounds scale in the same way: the chargedmeson component (B) increases whereas the other two decrease. Because the signal definition in Ref. [46] is different, the scaling factors for backgrounds (A) and (C) are significantly updated as expected. Details of the background fit can be found in Ref. [47]. The postfit distributions are shown in Fig. 4 for the reconstructed $p_{\mathrm{n}}$ and $\delta p_{\mathrm{TT}}$.

The reconstructed proton momentum resolution is improved by selecting elastically scattered and contained protons via an additional criterion that requires a large $\mathrm{d} E / \mathrm{d} x$ near the track end point [6]. As a result, this removes ill-determined momentum-by-range events whose selected protons either undergo inelastic scattering or are not contained in the tracker. This leads to a $p_{\mathrm{p}}$ resolution of $\sim 2 \%$ at $1 \mathrm{GeV} / c$ albeit at the cost of a $50 \%$ reduction in statistics.

The $\pi^{0}$ momentum reconstruction is improved via kinematic fitting [48,49]. Given the relationship between the $\pi^{0}$ mass and the photon kinematics in Eq. (14), the photon energies are recalculated by minimizing

$\chi^{2}=\left[\frac{m_{\pi}^{2}-2 E_{1} E_{2}\left(1-\cos \theta_{12}\right)}{\sigma_{m_{\pi}^{2}}}\right]^{2}+\sum_{i=1}^{2}\left[\frac{E_{i}-E_{i}^{\prime}}{\sigma\left(E_{i}\right)}\right]^{2}$,

where $E_{i}^{\prime}$ are the measured photon energies, and the recalculated energies, $E_{i}$, are treated as free parameters in the fit. The second term acts as a penalty for each photon and ensures that the fitted energies are within expectation of their calorimetrically measured values. Note that $\sigma_{m_{\pi}^{2}}$, representing the resolution of the reconstructed $\pi^{0}$ mass, is used as an optimization parameter whose value is chosen such that $99 \%$ of the fits successfully converge. The photon energy resolution, $\sigma\left(E_{i}\right)$, is determined from simulation. A full description can be found in Ref. [47]. This leads to a $\pi^{0}$ momentum resolution of about $20 \%$.
Flux-integrated cross sections are produced by first subtracting the constrained backgrounds from the selected samples. D'Agostini unfolding [50] is then performed with four iterations. The unfolding procedure is validated by reproducing pseudodata that is generated by extreme variations of the cross section models. The efficiency correction is then applied, followed by event normalization by the product of the flux and number of target nucleons $\left(3.12 \times 10^{30}\right)$. Systematic uncertainties are evaluated for all observables following Ref. [46]. In particular, parameters in the physics and detector models are varied within uncertainties and the resulting cross section variations are the assigned systematic uncertainties. For example, $p_{\mathrm{n}}$, whose statistical uncertainty spans $10 \%-34 \%$, has systematic uncertainties arising from detector (2\%-8\%), flux (3\%-8\%), and GENIE cross section models (5\%-28\%); as one of the GENIE model parameters, the aforementioned $M_{\mathrm{A}}^{\mathrm{QE}}$ leads to an uncertainty of $0.1 \%-1 \%$. The total uncertainty for $p_{\mathrm{n}}$ at few $\mathrm{MeV} / \mathrm{c}$ is approximately $22 \%$, increasing to $46 \%$ at $0.8 \mathrm{GeV} / c$. (See Supplemental Materials 1 and 2 [51] for details of cross section uncertainties.)

\section{RESULTS}

The measured cross section in $p_{\mathrm{n}}$ is compared to generator predictions in Fig. 5. The Fermi motion peak (below $0.25 \mathrm{GeV} / c$ ) is qualitatively captured by the NuWro (19.02) [52] RFG model. In this Fermi gas model, all nucleons lie below the Fermi surface and the predicted cross section in $p_{\mathrm{n}}$ has a cutoff at $0.22 \mathrm{GeV} / c$. For the previous QE-like measurement, the spectral function (SF) approach [53] best describes the data [6]. However, at present, while SF calculations for pion production exist $[54,55]$, they are not yet implemented in generators. In $\mathrm{NuWro}$, the effective spectral function (ESF) [56] incorporates the most important features of $\mathrm{SF}$ in generator implementation: in ESF, the probability distribution of the target nucleon momentum is identical to SF; for a selected value of the nucleon momentum, an average removal energy calculated from SF is used.

The nonexclusive part of the signal, such as multi- $\pi^{0}$ production, gives rise to large values of $p_{\mathrm{n}}$ beyond the Fermi surface and hence the long tail in the NuWro RFG prediction without FSIs. When FSIs are switched on, kinematic distortion migrates events away from the Fermi motion peak; pion absorption and charge exchange following multi- $\pi$ contributions and wrong-sign $\left(\Delta^{++}\right)$ production also add to the tail region. These IMT processes lead to a $p_{\mathrm{n}}$ tail that is similar in the RFG and ESF predictions. In this large-missing-pT region, NuWro with either initial-state models describes data within about $1-\sigma$. However, in the peak region, the data exhibit a distinctly muted distribution devoid of the sharp falloff. Furthermore, the ESF peak locates at around $0.15 \mathrm{GeV} / c, 25 \%$ off compared to data. 

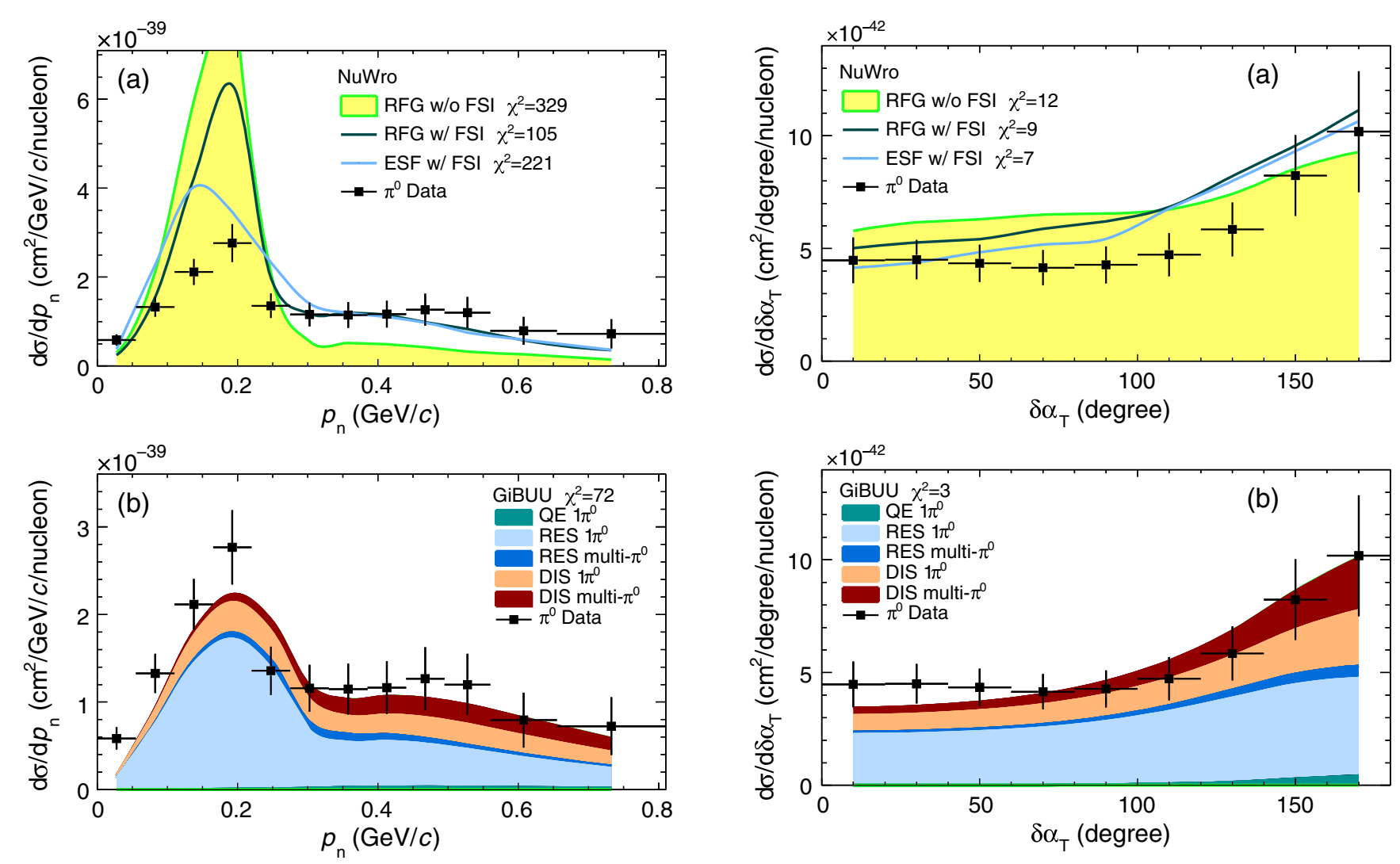

FIG. 5. Cross section in $p_{\mathrm{n}}$ compared to (a) NuWro 19.02 and (b) GiBUU 2019 predictions. Error bars on the data include both statistical and systematic uncertainties. The NuWro prediction for RFG without FSI has a maximum of $8.9 \times 10^{-39} \mathrm{~cm}^{2} / \mathrm{GeV} / c /$ nucleon. The GiBUU predictions are decomposed into single- and multi- $\pi^{0}$ contributions.

Comparison is also made to GiBUU (2019) [57-59] predictions in Fig. 5(b). While it also describes the largemissing-pT region, GiBUU underpredicts the Fermi motion peak. Nevertheless, it has the correct peak location and overall better describes the data. In GiBUU, the initial state is modeled as local Fermi gas in a nuclear potential [60]. Model features that decrease or enhance the exclusive proton- $\pi^{0}$ production will have as large an effect on the agreement as the initial state. From the decomposition of the interaction modes, it can be seen that besides the dominant resonant production, the DIS has a sizable contribution. Furthermore, the DIS contribution to the Fermi motion peak is dominated by single- $\pi^{0}$ production, and QE events wherein proton FSI initiates $\pi^{0}$ production give a small contribution.

The measured cross section in $\delta \alpha_{\mathrm{T}}$ is shown in Fig. 6 with model predictions. Because Fermi motion is isotropic, events in the $p_{\mathrm{n}}$ peak are evenly distributed in $\delta \alpha_{\mathrm{T}}$ and therefore provide a flat baseline for the overall cross section. The slope of the cross section toward $\delta \alpha_{\mathrm{T}}=$ $180^{\circ}$ comes from IMT events in the $p_{\mathrm{n}}$ tail. For the prediction without FSIs, the nonexclusive part of the signal

FIG. 6. Cross section in $\delta \alpha_{\mathrm{T}}$ compared to (a) NuWro 19.02 and (b) GiBUU 2019 predictions.

leads to the rise in cross section at large $\delta \alpha_{\mathrm{T}}$. With FSIs, the NuWro predictions become steeper and are much more similar with each other than in the case of $p_{\mathrm{n}}$-this is expected as NuWro FSI is decoupled from the initial state that gives a model-independent flat baseline. As NuWro overpredicts the $p_{\mathrm{n}}$ peak size with RFG and ESF, the overall predictions for $\delta \alpha_{\mathrm{T}}$ are above the data. For GiBUU, the slope is similar to that for NuWro, but the overall agreement is better because of the lower Fermi motion baseline.

In Fig. 7, the cross sections in $p_{\mathrm{n}}$ and $\delta \alpha_{\mathrm{T}}$ are area normalized and compared to those from the previous MINER $\nu \mathrm{A} C \mathrm{C}$ QE-like measurement [6],

$$
\nu_{\mu}+\mathrm{C} \rightarrow \mu^{-}+\mathrm{p}+\mathrm{X}^{\prime}
$$

where $\mathrm{X}^{\prime}$ is a final-state hadronic system consisting of the nuclear remnant with possible additional protons but without mesons. Both measurements are based on the same data set using the NuMI low energy neutrino beam. Similar to the discussions for Eqs. (1) and (9), in Eq. (16) when $X^{\prime}$ is carbon-11 and the final-state proton do not experience FSIs, $p_{\mathrm{n}}$ is the initial momentum of the struck neutron probed in the QE scattering,

$$
\nu_{\mu}+\mathrm{n} \rightarrow \mu^{-}+\mathrm{p}
$$



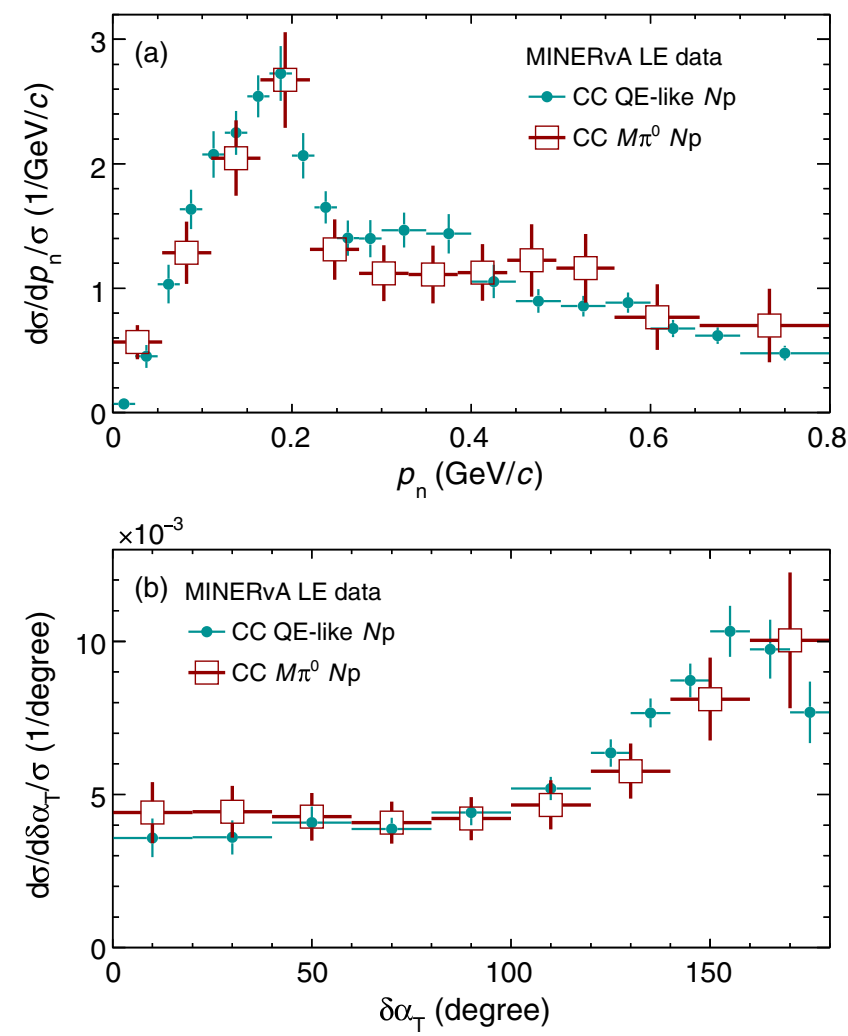

FIG. 7. Area-normalized cross sections in (a) $p_{\mathrm{n}}$ and (b) $\delta \alpha_{\mathrm{T}}$. Results from this work $\left(M \pi^{0} N \mathrm{p}\right)$ are compared to the previous MINER $\nu \mathrm{A} C \mathrm{CC}$ QE-like measurement [6]. Both measurements are based on the same data set using the NuMI low energy (LE) neutrino beam.

Comparing the shape of the $p_{\mathrm{n}}$ Fermi motion peak between the two measurements in Fig. 7(a), consistency is expected as the same initial state is probed. It is the consistency in the tail size (relative to the peak) that is nontrivial. In the largemissing-pT region, the underlying IMT processes are different: FSIs in QE, pion absorption in resonant production, and $2 \mathrm{p} 2 \mathrm{~h}$ on the one hand for Eq. (16) (see Ref. [6] and Supplemental Material 1 [51]), FSIs in resonant production, and DIS with or without pion absorption on the other hand for Eq. (1). Most notably, pion absorption increases the tail size in QE-like events, but decreases it in single-pion production.

The dynamics in the large-missing-pT region is further analyzed in the comparison of $\delta \alpha_{\mathrm{T}}$ in Fig. 7(b). Since the slope toward large $\delta \alpha_{\mathrm{T}}$ indicates how much impedance the outgoing hadrons experience inside the nucleus, the similar slopes in both measurements seem to suggest another possible fine-tuning: similar impedance in both QE-like and pion production, which is surprising because of the very different underlying IMT processes. While the consistency in both the $p_{\mathrm{n}}$-tail size and the large- $\delta \alpha_{\mathrm{T}}$ slope seem to be accidental, a combined analysis of both samples could provide very precise constraints on the modeling of the IMT processes. For completeness, GiBUU predictions
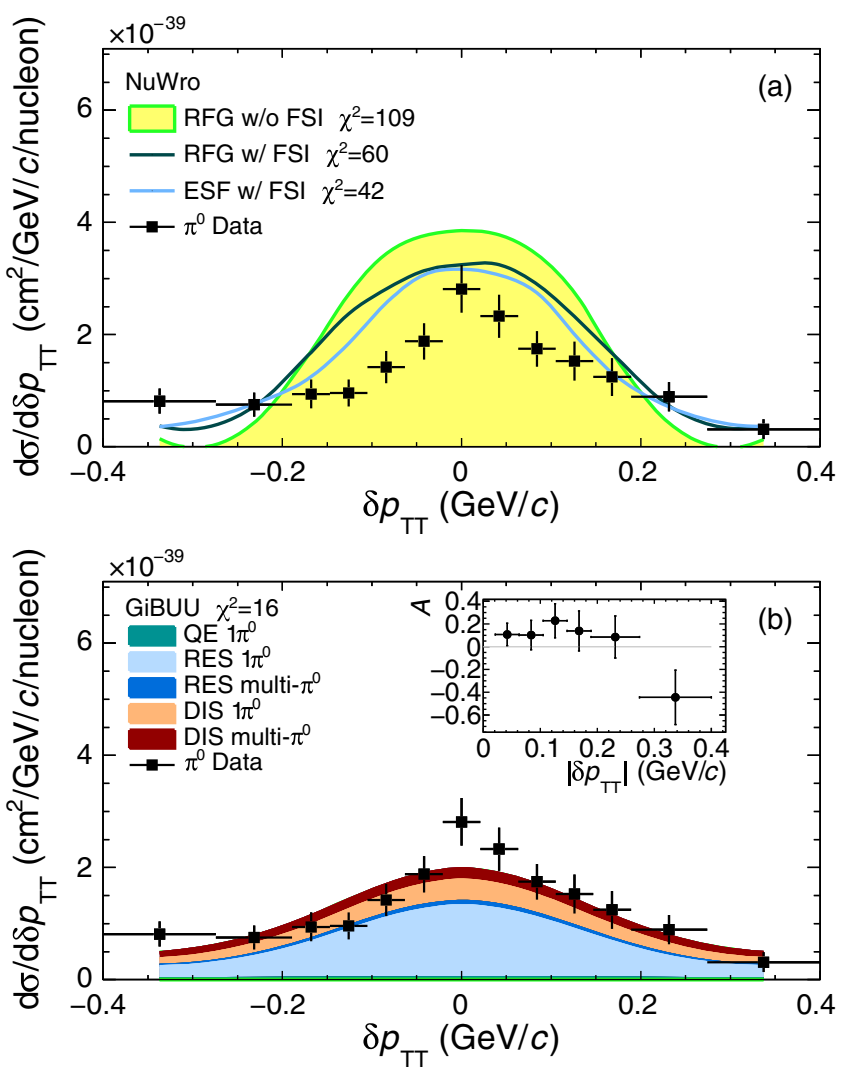

FIG. 8. Cross section in $\delta p_{\text {TT }}$ compared to (a) NuWro 19.02 and (b) GiBUU 2019 predictions. The $\delta p_{\mathrm{TT}}$ asymmetry, $A$ from Eq. (18), is shown as a function of $\left|\delta p_{\mathrm{TT}}\right|$ in the inset in (b).

for the QE-like measurement can be found in Supplemental Material 1 [51].

The cross section in $\delta p_{\mathrm{TT}}$ is shown in Fig. 8, compared to NuWro and GiBUU predictions. According to Eq. (10), part of the data-model discrepancy can be traced back to the mismodeling at the $p_{\mathrm{n}}$ peak as discussed above. The new aspect of nuclear effects probed by $\delta p_{\mathrm{TT}}$ is the symmetry of the cross section shape with respect to $\delta p_{\text {TT }}=0$. Without nuclear effects, the proton and $\pi^{0}$ momenta from Eq. (9) would be balanced with respect to the lepton scattering plane, yielding $\delta p_{\mathrm{TT}}=0$. Fermi motion and IMT contributions then introduce event-by-event imbalance to either side of the plane. Previous measurements including Ref. [46] indicate that the final-state $\pi^{0}$ could prefer one side of the scattering plane due to the interference between delta and nonresonant amplitudes (see Ref. [15] for discussions). Without loss of generality, suppose the proton in Eq. (1) would always prefer the positive- $\hat{z}_{\mathrm{TT}}$ side of the lepton scattering plane (Fig. 2). In such a case, we would have $\delta p_{\mathrm{TT}}=\left|\vec{p}_{\mathrm{p}} \cdot \hat{z}_{\mathrm{TT}}\right|-\left|\vec{p}_{\pi^{0}} \cdot \hat{z}_{\mathrm{TT}}\right|$. FSIs that change the proton and $\pi^{0}$ momenta differently could then cause a positive-negative asymmetry in $\delta p_{\text {TT }}$. In this measurement, the cross section asymmetries of each positivenegative $\delta p_{\mathrm{TT}}$-bin pair are shown in Fig. 8(b) inset. They are defined as 


$$
A\left(\left|\delta p_{\mathrm{TT}}\right|\right) \equiv \frac{\mathrm{d} \sigma\left(\left|\delta p_{\mathrm{TT}}\right|\right)-\mathrm{d} \sigma\left(-\left|\delta p_{\mathrm{TT}}\right|\right)}{\mathrm{d} \sigma\left(\left|\delta p_{\mathrm{TT}}\right|\right)+\mathrm{d} \sigma\left(-\left|\delta p_{\mathrm{TT}}\right|\right)}
$$

Compared to these observed mild asymmetries, negligible asymmetry is predicted by the generators.

\section{SUMMARY}

This paper presents the first measurements of a set of novel final-state correlations in neutrino $\mathrm{CC} \pi^{0}$ production. These TKI-based correlations include the transverse boosting angle $\left(\delta \alpha_{\mathrm{T}}\right)$ and the emulated nucleon momentum $\left(p_{\mathrm{n}}\right)$, both of which were previously measured only in CC QElike production $[6,10]$, as well as the double-transverse momentum imbalance $\left(\delta p_{\mathrm{TT}}\right)$ that has no previous measurement. This work separates Fermi motion and IMT in pion production for the first time, and the observed cross section shapes are consistent with the previous MINER $\nu \mathrm{A}$ measurement of QE-like interactions. In the present measurement, RFG and ESF models describe within $1-\sigma$ the large-missing-pT region $\left(p_{\mathrm{n}}>300 \mathrm{MeV} / c\right)$ that collectively comes from IMT processes including FSI distortions, nonexclusive contributions, and wrong-sign $\left(\Delta^{++}\right)$production. The measured exclusive proton- $\pi^{0}$ production cross section on neutrons in nuclei in the Fermi motion peak region $\left(p_{\mathrm{n}}<220 \mathrm{MeV} / c\right)$, however, is mismodeled by current generators using Fermi gases. Whereas spectral function can successfully describe the previous QE-like results, the effective spectral function approach still overpredicts the Fermi motion peak in pion production. New investigation of the final-state hadronic momentum configuration is made with $\delta p_{\mathrm{TT}}$, showing a mild asymmetry that could come from particle-type-dependent FSIs following interference effects in pion production.

In the future, nuclear effects associated with initial-state protons could be examined using proton- $\pi^{ \pm}$production in $\mathrm{CC} \stackrel{(-)}{\nu}_{\mu}$-nucleus scattering [13]. Measurements of proton$\pi^{0}$ systems as reported here could be performed in liquid argon TPC experiments such as ICARUS [61],
MicroBooNE [62], and SBND [61], providing clarifications of neutrino-argon interactions that are needed by the DUNE neutrino oscillation program. MINER $\nu$ As mediumenergy exposures allow new investigations at higher neutrino energies and with larger event samples [63,64]. Thus, further illumination of the physics that underwrites the TKI-based final-state correlations in $\mathrm{CC}$ pion production may be anticipated.

\section{ACKNOWLEDGMENTS}

We thank Luis Alvarez Ruso for helpful comments on the paper. This document was prepared by members of the MINER $\nu \mathrm{A}$ Collaboration using the resources of the Fermi National Accelerator Laboratory (Fermilab), a U.S. Department of Energy, Office of Science, HEP User Facility. Fermilab is managed by Fermi Research Alliance, LLC (FRA), acting under Contract No. DE-AC0207CH11359. These resources included support for the MINER $\nu \mathrm{A}$ construction project, and support for construction also was granted by the U.S. National Science Foundation under Grant No. PHY-0619727 and by the University of Rochester. Support for participating scientists was provided by NSF and DOE (USA); by CAPES and CNPq (Brazil); by CoNaCyT (Mexico); by Proyecto Basal FB 0821, CONICYT PIA ACT1413, Fondecyt 3170845 and 11130133 (Chile); by CONCYTEC (Consejo Nacional de Ciencia, Tecnologa e Innovacin Tecnolgica), DGIPUCP (Direccin de Gestin de la Investigacin-Pontificia Universidad Catlica del Peru), and VRI-UNI (ViceRectorate for Research of National University of Engineering) (Peru); and by the Latin American Center for Physics (CLAF); NCN Opus Grant No. 2016/21/B/ ST2/01092 (Poland); by Magdalen College Oxford and Science and Technology Facilities Council (UK). We thank the MINOS Collaboration for use of its near detector data. Finally, we thank the staff of Fermilab for support of the beam line, the detector, and computing infrastructure.
[1] L. Alvarez-Ruso et al., Prog. Part. Nucl. Phys. 100, 1 (2018).

[2] M. A. Acero et al. (NOvA Collaboration), Phys. Rev. Lett. 123, 151803 (2019).

[3] K. Abe et al. (T2K Collaboration), Nature (London) 580, 339 (2020); 583, E16 (2020).

[4] B. Abi et al. (DUNE Collaboration), arXiv:2002.03005.

[5] K. Abe et al. (Hyper-Kamiokande Collaboration), arXiv: 1805.04163.

[6] X. Lu et al. (MINERvA Collaboration), Phys. Rev. Lett. 121, 022504 (2018).
[7] T. Cai et al. (MINERvA Collaboration), Phys. Rev. D 101, 092001 (2020).

[8] X. G. Lu, L. Pickering, S. Dolan, G. Barr, D. Coplowe, Y. Uchida, D. Wark, M. Wascko, A. Weber, and T. Yuan, Phys. Rev. C 94, 015503 (2016).

[9] A. P. Furmanski and J. T. Sobczyk, Phys. Rev. C 95, 065501 (2017).

[10] K. Abe et al. (T2K Collaboration), Phys. Rev. D 98, 032003 (2018).

[11] S. Dolan, U. Mosel, K. Gallmeister, L. Pickering, and S. Bolognesi, Phys. Rev. C 98, 045502 (2018). 
[12] S. Dolan, arXiv: 1810.06043.

[13] X. Lu and J. T. Sobczyk, Phys. Rev. C 99, 055504 (2019).

[14] L. Harewood and R. Gran, arXiv:1906.10576.

[15] T. Cai, X. Lu, and D. Ruterbories, Phys. Rev. D 100, 073010 (2019).

[16] X.-G. Lu, D. Coplowe, R. Shah, G. Barr, D. Wark, and A. Weber, Phys. Rev. D 92, 051302 (2015).

[17] H. Duyang, B. Guo, S. Mishra, and R. Petti, arXiv: 1809.08752.

[18] H. Duyang, B. Guo, S. Mishra, and R. Petti, Phys. Lett. B 795, 424 (2019).

[19] L. Munteanu, S. Suvorov, S. Dolan, D. Sgalaberna, S. Bolognesi, S. Manly, G. Yang, C. Giganti, K. Iwamoto, and C. Jess-Valls, Phys. Rev. D 101, 092003 (2020).

[20] P. Hamacher-Baumann, X. Lu, and J. Martín-Albo, Phys. Rev. D 102, 033005 (2020).

[21] L. Aliaga et al. (MINERvA Collaboration), Phys. Rev. D 94, 092005 (2016); 95, 039903(A) (2017).

[22] L. Aliaga et al. (MINERvA Collaboration), Nucl. Instrum. Methods Phys. Res., Sect. A 743, 130 (2014).

[23] D. G. Michael et al. (MINOS Collaboration), Nucl. Instrum. Methods Phys. Res., Sect. A 596, 190 (2008).

[24] C. Andreopoulos et al., Nucl. Instrum. Methods Phys. Res., Sect. A 614, 87 (2010).

[25] A. Bodek and J. L. Ritchie, Phys. Rev. D 23, 1070 (1981).

[26] C. H. Llewellyn Smith, Phys. Rep. 3, 261 (1972).

[27] R. Bradford, A. Bodek, H. S. Budd, and J. Arrington, Nucl. Phys. B, Proc. Suppl. 159, 127 (2006).

[28] D. Rein and L. M. Sehgal, Ann. Phys. (N.Y.) 133, 79 (1981).

[29] A. Bodek, I. Park, and U.-k. Yang, Nucl. Phys. B, Proc. Suppl. 139, 113 (2005).

[30] H. Gallagher, Nucl. Phys. B, Proc. Suppl. 159, 229 (2006).

[31] C. Wilkinson, P. Rodrigues, S. Cartwright, L. Thompson, and K. McFarland, Phys. Rev. D 90, 112017 (2014).

[32] P. Rodrigues, C. Wilkinson, and K. McFarland, Eur. Phys. J. C 76, 474 (2016).

[33] B. Eberly et al. (MINERvA Collaboration), Phys. Rev. D 92, 092008 (2015).

[34] A. Bodek and U. K. Yang, J. Phys. G 29, 1899 (2003).

[35] T. Sjostrand, S. Mrenna, and P. Z. Skands, J. High Energy Phys. 05 (2006) 026.

[36] T. Yang, C. Andreopoulos, H. Gallagher, K. Hoffman, and P. Kehayias, Eur. Phys. J. C 63, 1 (2009).

[37] Z. Koba, H. B. Nielsen, and P. Olesen, Nucl. Phys. B40, 317 (1972).

[38] J. Nieves, I. Ruiz Simo, and M. J. Vicente Vacas, Phys. Lett. B 707, 72 (2012).

[39] J. T. Sobczyk, Phys. Rev. C 86, 015504 (2012).

[40] R. Gran, J. Nieves, F. Sanchez, and M. J. Vicente Vacas, Phys. Rev. D 88, 113007 (2013).

[41] J. Schwehr, D. Cherdack, and R. Gran, arXiv:1601.02038.
[42] P. A. Rodrigues et al. (MINERvA Collaboration), Phys. Rev. Lett. 116, 071802 (2016); 121, 209902(A) (2018).

[43] J. Nieves, J. E. Amaro, and M. Valverde, Phys. Rev. C 70, 055503 (2004); 72, 019902(E) (2005).

[44] S. A. Dytman and A. S. Meyer, AIP Conf. Proc. 1405, 213 (2011).

[45] S. Agostinelli et al. (GEANT4 Collaboration), Nucl. Instrum. Methods Phys. Res., Sect. A 506, 250 (2003).

[46] O. Altinok et al. (MINERvA Collaboration), Phys. Rev. D 96, 072003 (2017).

[47] D. Coplowe, Ph. D. thesis, Oxford University, 2018, FERMILAB-THESIS-2018-38.

[48] H. W. Baer et al., Nucl. Instrum. Methods 180, 445 (1981).

[49] H. Stroher, G. Koch, V. Metag, R. Beck, B. Schoch, and J. Vogt, Nucl. Instrum. Methods Phys. Res., Sect. A 269, 568 (1988).

[50] G. D’Agostini, Nucl. Instrum. Methods Phys. Res., Sect. A 362, 487 (1995).

[51] See Supplemental Materials 1 and 2 at http://link.aps.org/ supplemental/10.1103/PhysRevD.102.072007 for complementary model comparisons and numerical tables of cross sections and their uncertainties.

[52] T. Golan, C. Juszczak, and J. T. Sobczyk, Phys. Rev. C 86, 015505 (2012).

[53] O. Benhar, A. Fabrocini, S. Fantoni, and I. Sick, Nucl. Phys. A579, 493 (1994).

[54] H. Nakamura, M. Sakuda, T. Nasu, and O. Benhar, Phys. Rev. C 76, 065208 (2007).

[55] H. Nakamura, T. Nasu, M. Sakuda, and O. Benhar, AIP Conf. Proc. 981, 234 (2008).

[56] A. M. Ankowski and J. T. Sobczyk, Phys. Rev. C 74, 054316 (2006).

[57] T. Leitner, L. Alvarez-Ruso, and U. Mosel, Phys. Rev. C 73, 065502 (2006).

[58] T. Leitner, O. Buss, L. Alvarez-Ruso, and U. Mosel, Phys. Rev. C 79, 034601 (2009).

[59] O. Buss, T. Gaitanos, K. Gallmeister, H. van Hees, M. Kaskulov, O. Lalakulich, A. B. Larionov, T. Leitner, J. Weil, and U. Mosel, Phys. Rep. 512, 1 (2012).

[60] U. Mosel, J. Phys. G 46, 113001 (2019).

[61] M. Antonello et al. (MicroBooNE, LAr1-ND, and ICARUS-WA104 Collaborations), arXiv:1503.01520.

[62] C. Adams et al. (MicroBooNE Collaboration), Phys. Rev. D 99, 091102 (2019).

[63] E. Valencia et al. (MINERvA Collaboration), Phys. Rev. D 100, 092001 (2019).

[64] M. Carneiro et al. (MINERvA Collaboration), Phys. Rev. Lett. 124, 121801 (2020).

Correction: Two references and their citations in text were missing and have been inserted. 